\title{
Carbonatación del Hormigón Simposio Internacional RILEM
}

Prof. Dr. DEMETRIO GASPAR-TEBAR

IETcc

\section{INTRODUCCION}

El dióxido de carbono es capaz de reaccionar, en determinadas condiciones, con los compuestos anhidros o hidratados de los conglomerantes hidráulicos, dando lugar a otros compuestos que afectan en grado distinto al comportamiento de los conglomerados correspondientes. Al conjunto de reacciones que tienen lugar se conoce, en la química del cemento, con el nombre de carbonatación.

La reacción y velocidad de carbonatación de los conglomerantes hidráulicos dependen de gran número de variables, entre las que cabe destacar: tipo, composición mineralógica y características del conglomerante; cantidad de $\mathrm{CO}_{2}$, humedad relativa, temperatura y presión del medio en donde se encuentra el conglomerante o el conglomerado; edad del hormigón; relación agua/cemento; dosificación; compacidad; estado de hidratación del cemento; etc.

Los componentes anhidros e hidratados del cemento portland cuando reaccionan con el $\mathrm{CO}_{2}$ forman nuevos compuestos como son: los carbosilicatos, carboaluminatos y el carbonato cálcico (amorfo o cristalizado), en una primera etapa; finalmente, cuando la reacción es completa forman: carbonato cálcico y dióxido de silíceo hidratado, los silicatos cálcicos y carbonato cálcico, hidróxido de aluminio e hidróxido férrico, los aluminoferritos cálcicos, y desaparecen, por consiguiente, los silicatos y aluminatos correspondientes.

El carbonato cálcico, según las condiciones de la reacción, se puede encontrar amorfo o cristalizado como vaterita, aragonito y/o calcita, solos o en mezclas variables según el grado de evolución. El hidróxido de aluminio, amorfo en principio, se transforma en la forma cristalina metaestable bayerita y finalmente en gibsita.

La carbonatación del cemento anhidro afecta a la composición química y estructural del mismo y, por consiguiente, a sus propiedades físicas y fisicomecánicas. Así, por regla general, el grado de finura disminuye, a veces se produce un apelotonamiento, se incrementan la consistencia de la pasta y el tiempo de fraguado, disminuyen en grado considerable las resistencias mecánicas, sobre todo a compresión en las primeras edades, etc.

La carbonatación de los compuestos hidratados del cemento portland afecta, del mismo modo que en el caso del cemento anhidro, a la composición química y estructural de la pasta y, por lo tanto, a las características físicas y fisicomecánicas de los conglomerados correspondientes, influyendo en la durabilidad de las estructuras de hormigón. 
La carbonatación de la pasta hidratada de cemento reduce el $\mathrm{pH}$ del medio hasta valores inferiores a 9, próximos a 8 , por lo que si dicha reacción tiene lugar con la pasta que se encuentra junto a las armaduras se favorece la posibilidad de corrosión de las mismas.

La fijación del $\mathrm{CO}_{2}$ por los hormigones lleva consigo una disminución de la porosidad por la formación, especialmente, de $\mathrm{CaCO}_{3}$, a la vez que se produce una liberación de agua; de aquí, que según se realice la carbonatación se puede producir una fisuración del hormigón - hecho decisivo para la corrosión de las armaduras-, un incremento de la densidad, de la dureza superficial, de la estabilidad dimensional y, por regla general, de las resistencias mecánicas y de la resistencia química.

La carbonatación del hormigón puede realizarse naturalmente por el $\mathrm{CO}_{2}$ atmosférico o artificialmente cuando se quieren aprovechar las ventajas que produce dicho proceso en los hormigones.

\section{GRUPO DE TRABAJO “CARBONATACION” DE LA RILEM}

La importancia de las reacciones entre el $\mathrm{CO}_{2}$ y los compuestos de los conglomerantes hidráulicos anhidros o hidratados, así como la influencia que ejerce en las propiedades tecnológicas del hormigón, hizo que la RILEM crease en 1969 un Grupo de Trabajo presidido por el Dr. Venuat que tenía, fundamentalmente, los siguientes objetivos:

- Realizar una recopilación bibliográfica de los diversos trabajos publicados, así como de los trabajos en curso.

- Poner a punto los métodos de investigación y control de la carbonatación.

- Coordinar las investigaciones, reuniendo los diversos trabajos, examinando los diferentes parámetros considerados y estudiar los puntos comunes y divergentes.

- Progresar en la explicación científica de los fenómenos de carbonatación de los conglomerantes hidráulicos.

Líneas que, en gran parte, se han cumplido, habiendo recogido, además, las sugerencias de los miembros del grupo que se han estudiado y discutido con el mayor interés y celebrado un "Simposio Internacional" en Inglaterra.

\section{SIMPOSIO INTERNACIONAL RILEM: "CARBONATACION DEL HORIMIGON"}

Durante los días 5 y 6 de abril de 1976 se celebró en los locales de la Cement and Concrete Association (Wexham Springs, Inglaterra) el primer Simposio Internacional sobre carbonatación del hormigón al que asistieron 34 investigadores y técnicos representando a 15 países y en el que se presentaron 40 trabajos, que se comentaron ampliamente, agrupados en las seis directrices fundamentales que se citan a continuación, las cuales se desarrollaron en tres sesiones de trabajo:

Sesión A

Tema 1: Fisicoquímica de la carbonatación.

Tema 2: Medida de la carbonatación. 


\section{Sesión B}

Tema 3: Grado de carbonatación.

Tema 4: Carbonatación y sus efectos en las estructuras de hormigón. Propiedades tecnológicas.

\section{Sesión C}

Tema 5: Carbonatación, durabilidad y propiedades favorables de la carbonatación.

Tema 6: Carbonatación de hormigones especiales.

El Presidente del presente Simposio fue el Dr. Venuat del CERILH de Francia. Cada Sesión tuvo, a su vez, otro Presidente y cada Tema un Ponente General que füeron:

Sesión A: Dr. Halstead, de la Cement and Concrete Association de Inglaterra.

Sesión B: Dr. Wesche, del Institut für Bauforschung de Alemania Occidental.

Sesión CC: Prof. Dr. Lach, de la Universidad Técnica de Brno de Checoslovaquia.

Tema 1: Dr. Smolczyk, del Forschungsgemeinschaft Elsenhüttenschlacken de Alemania Occidental.

Tema 2: Dr. Forrester, de la Cement and Concrete Association de Inglaterra.

Tema 3: Dr. Alexandre, del CERILH de Francia.

Tema 4: Dr. Pihlajavaara, del Technical Research Centre de Finlandia.

Tema 5: Prof. Dr. Calleja, del Instituto Eduardo Torroja de la Construcción y del Cemento de España.

Tema 6: Dr. Vandenbosch, de Cimenteries CBR de Bélgica.

\section{Comunicaciones presentadas}

A continuación se incluye la relación de comunicaciones presentadas y autores de las mismas.

\section{Tema 1. Fisicoquímica de la carbonatación}

\section{Informe General}

1.1. Fenómenos físicos y químicos de la carbonatación.

H. G. Smolczyk.

\section{Comunicaciones}

1.2. Algunas observaciones sobre la carbonatación de soluciones de $\mathrm{Ca}(\mathrm{OH})_{2}$ y la fase líquida de la pasta de cemento. Mecanismo de la carbonatación del hormigón.

E. Koelliker. 
1.3. Acción del $\mathrm{CO}_{2}$ sobre el cemento portland anhidro.

D. Gaspar-Tébar, C. del Olmo-Rodríguez y T. Vázquez-Moreno.

1.4. Acción del $\mathrm{CO}_{2}$ sobre el cemento portland hidratado en condiciones normales.

D. Gaspar-Tebar, T. Vázquez-Moreno y M. Muñoz-Plaza.

1.5. Determinación de las modificaciones del $\mathrm{CaCO}_{3}$ en el hormigón carbonatado.

V. Lach y Z. Sauman.

1.6. Efecto del incremento de la temperatura de curado sobre la carbonatación del hormigón.

R. Krzywoblocka-Laurow.

1.7. Carbonatación de la pasta de cemento hidratada en contacto con calizas.

L. P. Kurasova y L. M. Larionova.

1.8. Naturaleza y estabilidad térmica de los productos de interacción entre los áridos calizos y ciertas fases del cemento $\left(\mathrm{C}_{3} \mathrm{~A}\right.$ y $\left.\mathrm{C}_{4} \mathrm{AF}\right)$.

A. Negro, M. Murat y L. Cussino.

\section{Tema 2. Medida de la carbonatación}

Informe General

2.1. Medida de la carbonatación

J. A. Forrester.

Comunicaciones

2.2. Dispositivo que permite seguir la carbonatación del hormigón in situ.

P. Longuet y B. Courtault.

2.3. Influencia de la carbonatación sobre la estructura de los poros de la pasta de cemento hidratada.

K. Essig y H. K. Hilsdorf.

2.4. Cambios en la porosidad del hormigón durante la carbonatación.

N. K. Rozental y S. N. Alekseev.

\section{Tema 3. Grado de carbonatación}

Informe General

3.1. Velocidad de carbonatación.

J. Alexandre. 


\section{Comunicaciones}

3.2. Exploraciones sobre el estudio alemán a largo plazo de la velocidad de carbonatación.

H. G. Smolczyk.

3.3. Modificaciones provocadas en la cinética de la carbonatación.

B. Thuret.

3.4. Comportamiento de morteros y hormigones que contienen cenizas volantes en la carbonatación.

P. Schubert y W. von Berg.

3.5. Comportamiento de morteros y hormigones hechos con "Jet Cement" (HSZ) en la carbonatación.

P. Schubert e Y. Efes.

3.6. Velocidad de carbonatación del hormigón.

S. N. Alekseev y. N. K. Rozental.

Tema 4. Carbonatación y sus efectos sobre las estructuras de hormigón. Propiedades tecnológicas

Informe General

4.1. Carbonatación, propiedades tecnológicas y efectos de la carbonatación sobre estructuras de hormigón.

S. E. Pihlajavaara.

\section{Comunicaciones}

4.2. Efecto de la carbonatación sobre las eflorescencias den superficies de hormigón.

P. Samuelsson.

4.3. Influencia de la carbonatación asociada al tratamiento acelerado sobre el color superficial del hormigón.

F. Dutruel y R. Guyader.

4.4. La carbonatación del cemento aluminoso.

H. G. Midgley y B. M. Woodward.

4.5. Un estudio experimental del efecto de la carbonatación sobre la resistencia del hormigón.

S. E. Pihlajavaara.

4.6. Sobre el efecto de la carbonatación en la retracción y cambio de peso del hormigón. S. E. Pihlajavaara. 
4.7. Carbonatación de morteros y hormigones de cemento blanco y coloreado.

M. Enculescu.

\section{Tema 5. Carbonatación, durabilidad y propiedades favorables de la carbonatación}

\section{Informe General}

5.1. A propósito de los aspectos favorables de la carbonatación del hormigón.

J. Calleja.

Comunicaciones

5.2. Carbonatación acelerada y resistencias mecánicas.

H. Hornain.

5.3. Carbonatación acelerada y resistencia de los cementos a las aguas agresivas.

M. Regourd.

5.4. Acción del $\mathrm{CO}_{2}$ sobre pastas puras y sobre morteros (1:2) elaborados con diferentes cementos españoles.

O. Fernández-Peña.

5.5. Relación entre la carbonatación del cemento portland, el grado de cocción del clínker y algunos de los fenómenos expansivos del ensayo de autoclave.

A. Ruiz de Gauna.

5.6. Carbonatación de hormigones de cemento aluminoso.

E. Raask.

\section{Tema 6. Carbonatación de hormigones especiales}

Informe General

6.1. La carbonatación de los hormigones especiales.

V. Vandenbosch.

Comunicaciones

6.2. Comportamiento de hormigones de poliestireno expandido en la carbonatación. V. Hermann, P. Schubert y K. Wesche.

6.3. Sobre la carbonatación a largo plazo de los componentes de la pasta en hormigones celulares.

Z. Sauman y V. Lach. 
6.4. Carbonatación de hormigones celulares bajo las condiciones en que se encuentran expuestas las estructuras.

M. Matousek.

6.5. Carbonatación de hormigones de áridos ligeros.

V. F. Stepanova y S. N. Alekseev.

6.6. Carbonatación de morteros que endurecen en contacto de cerámica porosa.

J. Grandet y A. Vaquier.

6.7. Comportamiento de los hormigones calorifugados y de estructuras en presencia de gas carbónico a presión, a diversas temperaturas.

P. Mauny y A. Bernard.

6.8. Algunas consideraciones sobre la carbonatación y sobre la durabilidad de los hormigones ligeros resistentes.

I. Ionescu.

6.9. Sobre el mecanismo de la carbonatación del aluminato cálcico hexahidrato en el cemento aluminoso hidratado.

A. Ruiz de Gauna, F. Triviño Vázquez y T. Vázquez Moreno. 